\title{
SOME DUAL INTEGRAL EQUATIONS INVOLVING INVERSE FINITE MELLIN TRANSFORMS
}

\author{
by JOHN TWEED
}

(Received 9 March, 1972)

1. Introduction. The object of this paper is to find the solutions of some dual equations involving the inverses of certain Mellin type transforms that were first introduced by $D$. Naylor in his paper [1]. Because these transforms are relatively unknown we shall begin by defining them and making a note of some of their properties. The main result is contained in the following theorem.

THEOREM. Let $y^{c-1} f(y) \in L(0, R)$ for every real number $c$ such that $|c|<\sigma$ and let $f(y)$ be of bounded variation in the neighbourhood of the point $y=x \in(0, R)$. Let

$$
f^{ \pm}(s)=\int_{0}^{R}\left\{x^{s-1} \pm R^{2 s} x^{-s-1}\right\} f(x) d x \quad(s=c+i b) .
$$

Then

$$
\frac{1}{2}\{f(x+0)+f(x-0)\}=\frac{1}{2 \pi i} \int_{c-i \infty}^{c+i \infty} f^{ \pm}(s) x^{-s} d s \quad(|c|<\sigma) .
$$

Proof. In order to prove this theorem, we introduce the functions $g^{ \pm}(x)$ which are defined by the equations

$$
g^{ \pm}(x)=\left\{\begin{array}{ll}
f(x) & (0<x<R) \\
\pm f\left(R^{2} / x\right) & (R<x<\infty)
\end{array}\right\}
$$

The result now follows from the facts that the functions $g^{ \pm}(x)$ satisfy the conditions of the Mellin inversion theorem (see [2]) and that

$$
\mathscr{M}\left[g^{ \pm}(x) ; s\right]=f^{ \pm}(s) \quad(|\operatorname{Re}(s)|<\sigma) .
$$

We now introduce the finite Mellin transforms $M_{R}[f(x) ; s]$ and $N_{R}[f(x) ; s]$, which are defined by the equations

$$
M_{R}[f(x) ; s]=\int_{0}^{R}\left[x^{s-1}+R^{2 s} x^{-s-1}\right] f(x) d x
$$

and

$$
N_{R}[f(x) ; s]=\int_{0}^{R}\left[x^{s-1}-R^{2 s} x^{-s-1}\right] f(x) d x,
$$

respectively. Clearly, if $f(x)$ satisfies the conditions of the theorem above, then the inversion 
formulae for these transforms are given by (1.2). The following properties of the transforms are easily obtained from the definitions.

1. $M_{R}\left[x \frac{d f}{d x} ; s\right]=2 R^{s} f(R)-s N_{R}[f ; s]$.

2. $N_{R}\left[x \frac{d f}{d x} ; s\right]=-s M_{R}[f ; s]$.

3. $M_{R}\left[\left(x \frac{d}{d x}\right)^{2} f(x) ; s\right]=s^{2} M_{R}[f ; s]+2 R^{s+1}\left(\frac{d f}{d x}\right)_{R}$.

4. $N_{R}\left[\left(x \frac{d}{d x}\right)^{2} f(x) ; s\right]=s^{2} N_{R}[f ; s]-2 R^{s} s f(R)$.

5. $M_{R}[f(u x) ; s]=u^{-s} M_{R u}[f(x) ; s] \quad(u>0)$.

$$
N_{R}[f(u x) ; s]=u^{-s} N_{R u}[f(x) ; s] \quad(u>0) .
$$

6. $M_{R}\left[f\left(x^{n}\right) ; s\right]=n^{-1} M_{R^{n}}[f(x) ; s / n] \quad(n>0)$.

$$
N_{R}\left[f\left(x^{n}\right) ; s\right]=n^{-1} N_{R^{n}}[f(x) ; s / n] \quad(n>0) .
$$

7. $M_{R}[H(x-t) ; s]=s^{-1}\left[R^{2 s} t^{-s}-t^{s}\right] \quad(0<t<R)$.

8. $N_{R}[H(x-t) ; s]=-s^{-1} t^{-s}\left(R^{s}-t^{s}\right)^{2} \quad(0<t<R)$.

9. $M_{R}\left[\frac{R^{2 n}}{R^{2 n}-x^{n} t^{n}}-\frac{t^{n}}{t^{n}-x^{n}} ; s\right]=\frac{\pi}{n}\left(R^{2 s} t^{-s}-t^{s}\right) \cot \frac{\pi s}{n} \quad(|\operatorname{Re}(s)|<n)$.

10. $N_{R}\left[\frac{(R x)^{n / 2}}{x^{n}-R^{n}} ; s\right]=\frac{\pi R^{s}}{n} \tan \frac{\pi s}{n} \quad\left(|\operatorname{Re}(s)|<\frac{1}{2} n\right)$.

2. Some dual equations involving $M_{R}^{-1}$. In this section we consider the dual equations

$$
\left.\begin{array}{cc}
M_{R}^{-1}\left[s^{-1} A(s) ; x\right]=0 & (0<x<a), \\
M_{R}^{-1}\left[A(s) \cot \frac{\pi s}{n} ; x\right]=f(x) & (a<x<R),
\end{array}\right\}
$$

where $|\operatorname{Re}(s)|<n$. In order to solve these equations, we begin by assuming that $A(s)$ may be written in the form

$$
A(s)=\int_{a}^{R} t^{n-1} p\left(t^{n}\right)\left(R^{2 s} t^{-s}-t^{5}\right) d t
$$


With this choice of $A(s)$, we find that

$$
\begin{aligned}
M_{R}^{-1}\left[s^{-1} A(s) ; x\right] & =\int_{a}^{R} t^{n-1} p\left(t^{n}\right) M_{R}^{-1}\left[s^{-1}\left(R^{2 s} t^{-s}-t^{s}\right) ; x\right] d t \\
& =\left\{\begin{array}{ll}
0 & (0<x<a), \\
\int_{a}^{x} t^{n-1} p\left(t^{n}\right) d t & (a<x<R),
\end{array}\right\}
\end{aligned}
$$

so that the first of the equations (2.1) is satisfied automatically. Similarly, we see that

$$
M_{R}^{-1}\left[A(s) \cot \frac{\pi s}{n} ; x\right]=\int_{a}^{R} t^{n-1} p\left(t^{n}\right) M_{R}^{-1}\left[\left(R^{2 s} t^{-s}-t^{s}\right) \cot \frac{\pi s}{n} ; x\right] d t
$$

and hence that the second dual equation will be satisfied if

$$
\frac{1}{\pi} \int_{a}^{R} n t^{n-1} p\left(t^{n}\right)\left\{\frac{R^{2 n}}{R^{2 n}-x^{n} t^{n}}-\frac{t^{n}}{t^{n}-x^{n}}\right\} d t=f(x) \quad(a<x<R)
$$

If we now make the change of variable $\tau=(t / R)^{n}$, let $b=(a / R)^{n}$ and $q(\tau)=R^{n} p\left(R^{n} \tau\right)$ and put $\rho$ equal to $(x / R)^{n}$ and then $(R / x)^{n}$ in turn, we find that (2.4) implies that

$$
\frac{1}{\pi} \int_{b}^{1} q(\tau)\left\{\frac{\tau}{1-\rho \tau}-\frac{1}{\tau-\rho}\right\} d \tau= \begin{cases}\rho^{-1} f\left(R \rho^{1 / n}\right) & (b<\rho<1), \\ \rho^{-1} f\left(R \rho^{-1 / n}\right) & \left(1<\rho<b^{-1}\right),\end{cases}
$$

that is,

$$
\frac{1}{\pi} \int_{1}^{b^{-1}} \frac{\tau^{-2} q\left(\tau^{-1}\right)}{\tau-\rho} d \tau-\frac{1}{\pi} \int_{b}^{1} \frac{q(\tau)}{\tau-\rho} d \tau=\left\{\begin{array}{ll}
\rho^{-1} f\left(R \rho^{1 / n}\right) & (b<\rho<1), \\
\rho^{-1} f\left(R \rho^{-1 / n}\right) & \left(1<\rho<b^{-1}\right) .
\end{array}\right\}
$$

By writing

$$
h(\tau)=\left\{\begin{array}{ll}
-q(\tau) & (b<\tau<1), \\
\tau^{-2} q\left(\tau^{-1}\right) & \left(1<\tau<b^{-1}\right),
\end{array}\right\} \text { and } g(\rho)=\left\{\begin{array}{ll}
\rho^{-1} f\left(R \rho^{1 / n}\right) & (b<\rho<1), \\
\rho^{-1} f\left(R \rho^{-1 / n}\right) & \left(1<\rho<b^{-1}\right),
\end{array}\right\}
$$

we find that $(2.5)$ takes the form

$$
\frac{1}{\pi} \int_{0}^{b^{-1}} \frac{h(\tau) d \tau}{\tau-\rho}=g(\rho) \quad\left(b<\rho<b^{-1}\right),
$$

which is an integral equation whose solution (see Tricomi [3]) is given by

$$
h(\tau)=\frac{1}{\Delta(\tau)}\left\{C-\frac{1}{\pi} \int_{b}^{b^{-1}} \frac{\Delta(y)}{y-\tau} g(y) d y\right\}
$$


where $\Delta(\tau)=\left[\left(b^{-1}-\tau\right)(\tau-b)\right]^{1 / 2}$ and $C$ is an arbitrary constant. In order to determine the constant $C$, we make use of the fact that $h\left(\tau^{-1}\right)=-\tau^{2} h(\tau)$ and $g\left(\rho^{-1}\right)=\rho^{2} g(\rho)$, from which it follows that $C=0$ and hence that

$$
h(\tau)=-\frac{1}{\pi \Delta(\tau)} \int_{b}^{b-1} \frac{\Delta(y)}{y-\tau} g(y) d y .
$$

It is now a simple matter to show that $p\left(t^{n}\right)$ is given by the formula

$$
\begin{aligned}
p\left(t^{n}\right)= & \frac{n}{\pi\left[\left(R^{2 n}-a^{n} t^{n}\right)\left(t^{n}-a^{n}\right)\right]^{1 / 2}} \\
& \times \int_{a}^{R}\left[\left(R^{2 n}-a^{n} x^{n}\right)\left(x^{n}-a^{n}\right)\right]^{1 / 2}\left\{\frac{1}{x^{n}-t^{n}}+\frac{R^{n}}{R^{2 n}-x^{n} t^{n}}\right\} \frac{f(x)}{x} d x .
\end{aligned}
$$

The solution of the dual equations may now be obtained by substituting from (2.9) into (2.2).

3. Some dual equations involving $N_{R}^{-1}$. We now turn our attention to the dual equations

$$
\left.\begin{array}{ll}
N_{R}^{-1}\left[s^{-1} A(s) ; x\right]=0 & (0<x<a), \\
N_{R}^{-1}\left[A(s) \tan \left(\frac{\pi s}{n}\right) ; x\right]=f(x) & (a<x<R),
\end{array}\right\}
$$

where $|\operatorname{Re}(s)|<\frac{1}{2} n$. In order to solve these equations, we assume that $A(s)$ may be written in the form

where

$$
A(s)=\int_{a}^{R} t^{\frac{1 n-s-1}{n-1}} p\left(t^{n}\right)\left(R^{s}-t^{s}\right)^{2} d t,
$$

$$
\int_{a}^{R} t^{t n-1} p\left(t^{n}\right) d t=0
$$

In this case we see that

$$
\begin{aligned}
N_{\mathrm{R}}^{-1}\left[s^{-1} A(s) ; x\right] & =\int_{a}^{R} t^{\frac{t n-s-1}{2 n}\left(t^{n}\right) N_{\mathrm{R}}^{-1}\left[s^{-1}\left(R^{s}-t^{5}\right)^{2} t^{-s} ; x\right] d t} \\
& =\left\{\begin{array}{ll}
-\int_{a}^{x} t^{\frac{1}{n} n-1} p\left(t^{n}\right) d t & (a<x<R), \\
0 & (0<x<a),
\end{array}\right\}
\end{aligned}
$$

and, as before, we find that the first dual equation is satisfied automatically. Similarly, we find that

$$
\begin{aligned}
N_{R}^{-1}\left[A(s) \tan \frac{\pi s}{n} ; x\right] & =\int_{a}^{R} t^{\frac{1}{n-1}} p\left(t^{n}\right) N_{R}^{-1}\left[\frac{\left(R^{s}-t^{s}\right)^{2}}{t^{s}} \tan \frac{\pi s}{n} ; x\right] d t \\
& =-\frac{n x^{\frac{1}{2 n}}}{\pi R^{n}} \int_{a}^{R} t^{n-1} p\left(t^{n}\right)\left\{\frac{1}{1-\left(x t / R^{2}\right)^{n}}+\frac{1}{(t / R)^{n}-(x / R)^{n}}\right\} d t,
\end{aligned}
$$


so that the second dual equation will be satisfied if

$$
\frac{n}{\pi R^{n}} \int_{a}^{R} t^{n-1} p\left(t^{n}\right)\left\{\frac{1}{1-\left(x t / R^{2}\right)^{n}}+\frac{1}{(t / R)^{n}-(x / R)^{n}}\right\} d t=-\frac{f(x)}{x^{t^{n}}} \quad(a<x<R) .
$$

If we now make the change of variable $\tau=(t / R)^{n}$ and then put $\rho$ equal to $(x / R)^{n}$ and $(R / x)^{n}$ in turn we find, as in the previous section, that this equation may be written in the form

$$
\frac{1}{\pi} \int_{b}^{b^{-1}} \frac{h(\tau)}{\tau-\rho} d \tau=g(\rho) \quad\left(b<\rho<b^{-1}\right)
$$

where $b=(a / R)^{n}$,

$$
h(\tau)=\left\{\begin{array}{ll}
p\left(R^{n} \tau\right) & (b<\tau<1), \\
\tau^{-1} p\left(R^{n} \tau^{-1}\right) & \left(1<\tau<b^{-1}\right)
\end{array}\right\}
$$

and

$$
g(\rho)=\left\{\begin{array}{cc}
-f\left(R \rho^{1 / n}\right) /\left(R^{n} \rho\right)^{1 / 2} & (b<\rho<1), \\
f\left(R \rho^{-1 / n}\right) /\left(R^{n} \rho\right)^{1 / 2} & \left(1<\rho<b^{-1}\right)
\end{array}\right\}
$$

The solution of equation (3.5) is given by

$$
h(\tau)=\frac{1}{\Delta(\tau)}\left\{C-\frac{1}{\pi} \int_{b}^{b^{-1}} \frac{\Delta(y) g(y)}{y-\tau} d y\right\}
$$

where $\Delta(\tau)=\left[\left(b^{-1}-\tau\right)(\tau-b)\right]^{1 / 2}$ and $C$ is an arbitrary constant. In order to determine $C$, we must apply the condition (3.3), which may be written in the form

$$
\int_{b}^{b-1} \tau^{-1 / 2} h(\tau) d \tau=0
$$

On applying this condition, we discover that $C$ is given by

$$
C=\frac{1}{2 \pi F b^{1 / 2}} \int_{b}^{b^{-1}} \frac{d \tau}{\Delta(\tau) \tau^{1 / 2}} \int_{b}^{b^{-1}} \frac{\Delta(y) g(y)}{y-\tau} d y,
$$

where $F$ is the complete elliptic integral $F\left(\frac{1}{2} \pi,\left[1-b^{2}\right]^{1 / 2}\right)$. After a little manipulation it now follows that

$$
p\left(t^{n}\right)=a^{ \pm n}\left[\left(R^{2 n}-a^{n} t^{n}\right)\left(t^{n}-a^{n}\right)\right]^{-\frac{1}{t}}\left\{\frac{n R^{n}}{F} \int_{a}^{R} \frac{s^{\frac{1 n-1}{}} I\left(s^{n}\right) d s}{\left[\left(R^{2 n}-a^{n} s^{n}\right)\left(s^{n}-a^{n}\right)\right]^{ \pm}}-I\left(t^{n}\right)\right\},
$$

where

$$
I\left(t^{n}\right)=\frac{n t^{n}}{\pi a^{\frac{1}{2 n}}} \int_{a}^{R} \frac{\left[\left(R^{2 n}-a^{n} x^{n}\right)\left(x^{n}-a^{n}\right)\right]^{\frac{1}{2}}}{\left(x^{n}-t^{n}\right)\left(R^{2 n}-t^{n} x^{n}\right)} \frac{\left(x^{2 n}-R^{2 n}\right) f(x)}{x^{\frac{1}{2 n+1}}} d x .
$$

The solution $A(s)$ of the dual equations (3.1) may now be obtained by substituting from (3.9) and (3.10) into (3.2). 


\section{REFERENCES}

1. D. Naylor, On a Mellin type of integral transform, J. Math. Mech. 12 (1963), 265-274.

2. E. C. Titchmarsh, Theory of Fourier Integrals (Oxford, 1937).

3. F. A. Tricomi, On the finite Hilbert transformation, Quart. J. Math. Oxford Ser. (2) 2 (1951), 199-211.

\section{UNIVERSITY OF GLASGOW \\ Glasgow G12 8QQ}

